\title{
A Texture Manifold for Curve-Based Morphometry of the Cerebral Cortex
}

\author{
Maxime Boucher ${ }^{\star}$ Alan Evans, and Kaleem Siddiqi \\ Center for Intelligent Machines, McGill University \\ \{boucher, siddqi\}@cim.mcgill.ca \\ McConnell Brain Imaging Center, McGill University \\ \{alan\}@bic.mni.mcgill.ca
}

\begin{abstract}
The cortical surface of the human brain is composed of folds that are juxtaposed alongside one another. Several methods have been proposed to study the shape of these folds, e.g., by first segmenting them on the cortical surface or by analysis via a continuous deformation of a common template. A major disadvantage of these methods is that, while they can localize shape differences, they cannot easily identify the directions in which they occur. The type of deformation that causes a fold to change in length is quite different from that which causes it to change in width. Furthermore, these two deformations may have a completely different biological interpretation. In this article we propose a method to analyze such deformations using directional filters locally adapted to the geometry of the folding pattern. Motivated by the texture flow literature in computer vision we recover flow fields that maintain a fixed angle with the orientation of folds, over a significant spatial extent. We then trace the flow fields to determine which correspond to the shape changes that are the most salient. Using the OASIS database, we demonstrate that in addition to known regions of atrophy, our method can find subtle but statistically significant shape deformations.
\end{abstract}

\section{Introduction}

The human cortical surface is composed of a set of folds that run alongside one another to form an undulating pattern of crests and troughs. The shape of this folding pattern evolves during the normal cycle of human brain development under the effect of aging or the presence of diseases or cognitive deficits. A popular method to capture shape differences is to view brain development as a continuous deformation of a common template [1-3]. The continuous deformation between the common template and individual cortical surfaces is typically constrained to be a diffeomorphism and is obtained using surface registration techniques. Deformation statistics are then used to find regions of significant tissue growth or atrophy. Such statistics can determine, for example, if the overall area of a particular region is larger in one group compared with another.

\footnotetext{
* Corresponding author: boucher@cim.mcgill.ca
} 
In addition to diffeomorphism based statistics, which capture local changes in shape, regularization filters such as the isotropic diffusion kernel on surfaces $[4,5]$, can yield a more global measure of shape differences. However, anatomical structures in the human brain are typically not isotropic and nor are the changes they induce on the cortical surface as they deform. As an example Fig. 1 illustrates two distinct deformations of a folding pattern, represented by a set of parallel curves. In the context of cortical shape analysis it is therefore beneficial to analyze differences using filters that are tuned to specific orientations. A neuroscientist can then examine whether an observed shape difference in a group follows a particular direction relative to fold orientation.

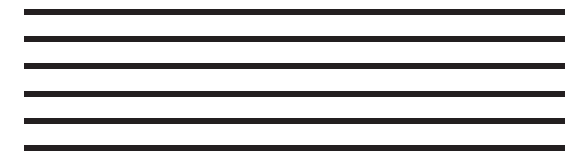

(a)

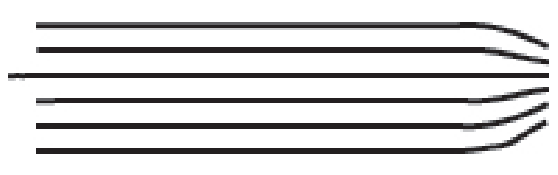

(b)

Fig. 1. Fig. 1(b) shows two deformations of the folding pattern in Fig. 1(a). On Fig. 1(b), a deformation parallel to a fold increases its length, and perpendicular to folds decreases the spacing between folds.

In this paper we abstract the shape of cortical folds as a collection of juxtaposed curves that locally have similar orientations. We then design shape filters which maintain a constant angle with neighboring folds. For example, a particular shape filter can be designed to be sensitive to a deformation parallel to fold orientation (an angle of 0 degrees), while another can be sensitive to a deformation that is perpendicular. This permits an analysis which is relative to cortical fold orientation. The challenge here is the estimation of the curvature to apply so that each filter maintains this constant angle.

To illustrate the computation of curvature consider the fingerprint example of Fig. 2(a), which is a pattern formed of juxtaposed intensity ridges similar to the folds of the cerebral cortex. The output of a directional ridge detector applied to the slice in blue is shown in Fig. 2(b), as an intensity function of angle $(\theta)$ versus position $x_{1}$. It is evident that this output aggregates along a a continuous curve (or submanifold), dubbled a texture flow in the work of [6]. When the exact location of the submanifold of maximum intensity in Fig. 2(b) is known the submanifold can be characterized as a function of orientation in space $\Theta\left(x_{1}, x_{2}\right)$. Here $\Theta$ gives the orientation of neighboring curves at a location $\left(x_{1}, x_{2}\right)$ of Fig. 2(a). The curvature of neighboring curves is expressed as the gradient of $\Theta$ as $\nabla \Theta$. On the other hand, the slope of the aggregated intensity curve in Fig. 2(b) is also given by $\nabla \Theta$. Our aim in this paper is to determine the slope of the texture flow, and then generate directional filters using the curvature implied by this slope.

Inspired by the work of [6] we achieve a globally optimal assignment of curvatures to the cortical surface by using a dictionary of smooth flow fields on the surface. Each smooth flow field provides a hypothesis for the slope of the texture 


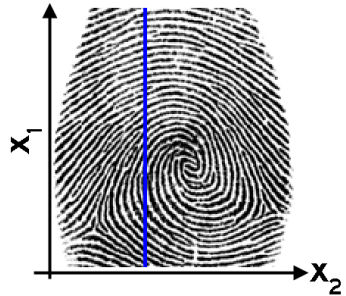

(a) Fingerprint image

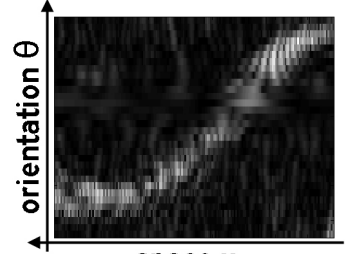

space $x_{1}$

(b) Ridge detection on Fig. 2(a) along the blue slice

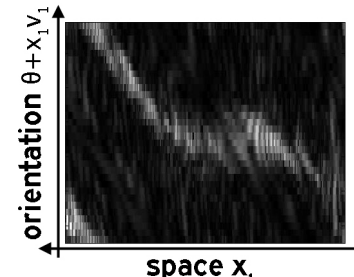

(c) Fig. 2(b) resampled along a given slope $v_{1}$.

Fig. 2. A texture manifold depicts change in orientation of the neighboring curves in space

flow on the cerebral cortex. We measure the goodness of fit between each flow field and the actual texture manifold by using anisotropic filters. We then use relaxation labeling to achieve a globally optimum assignment of smooth flow fields that match the cortical folds on the surface. Directional filters are then generated by launching streamlines whose curvature is provided by this assignment.

Using the OASIS database [7] we test our framework to determine how the shape of cortical folds in patients with cognitive impairment is affected. We are able to identify folds that undergo significant deformation in the temporal lobe, and also a stretch in length of the Cingulate gyrus below the Praecuneus area and below the sup frontal cortex in the left hemisphere. These results are also partially revealed by a statistical analysis based on surface area (see Fig. 5). However, our results clearly indicate that the increase in surface area comes from a stretch in sulcal length (see Fig. 6(b)). Whether this stretch in length is due to structural connectivity loss in the white matter remains to be investigated, but our findings are corroborated by a visual inspection of shape differences between the two groups.

\section{Estimating the Texture Manifold from Folds}

We give a brief technical overview of the algorithm before detailing each step. We refer again to Figure 2 to illustrate the method. Suppose that the output of the ridge detection in Figure 2(a) can be expressed as an intensity function $I\left(x_{1}, x_{2}, \theta\right) \rightarrow \mathbb{R}^{+}$. We formulate different hypotheses $H_{i}$ about the slope of the texture flow. Suppose, for example, that one such hypothesis $H_{i}$ states that the texture flow has a slope of $\left(v_{1}, v_{2}\right)$. This means that if we resample $I$ as

$$
I_{i}\left(x_{1}, x_{2}, \theta\right)=I\left(x_{1}, x_{2}, \theta+v_{1} x_{1}+v_{2} x_{2}\right),
$$

then, if this hypothesis is good, the intensity function should aggregates along an "horizontal" plane (as shown in Fig. 2(c)). A simple way to measure if a particular hypothesis is a good explanation for the observed texture flow is to measure how horizontal it is using an anisotropic filter. Suppose that $g_{a, b}$ is a Gaussian kernel where $a$ expresses the width of the kernel along the spatial 
dimensions $\left(x_{1}, x_{2}\right)$ and $b$ expresses the width of the kernel along the orientation dimension $\theta$ as

$$
g_{a, b}\left(x_{1}, x_{2}, \theta\right)=\left((2 \pi)^{3 / 2} a^{2} b\right)^{-1} \exp \left(-\frac{x_{1}^{2}+x_{2}^{2}}{2 a^{2}}-\frac{\theta^{2}}{2 b^{2}}\right) .
$$

A good measure to determine if $H_{i}$ is a good hypothesis is

$$
f_{i}\left(x_{1}, x_{2}, \theta\right)=\left(\frac{\partial}{\partial \theta} I_{i} * g_{a, b}\right)^{2}
$$

where $*$ is a convolution. The best hypothesis $H_{i}$ is the one that locally maximizes Equation 3. However, an optimal hypothesis should be a good fit for several curves in a large neighborhood. We therefore use each hypothesis $H_{i}$ as labels in the space formed by $\left(x_{1}, x_{2}, \theta\right)$. We achieve a globally optimum labeling $H^{*}\left(x_{1}, x_{2}, \theta\right)$ by selecting, at each point, the label which is both a good fit to the intensity function $I$ and is the most similar to the neighboring labels.

The ridge detector for surfaces is presented in Section 2.1, the algorithm to generate flow hypotheses $H_{i}$ on surfaces is explained in Section 2.2 and the relaxation labeling approach to find an optimal assignment is explained in Section 2.3. Once an optimal assignment is reached, it is possible to generate streamlines that follow the geometry of the folding pattern, which can then be used to detect shape changes. The algorithm to generate streamlines and use them to test statistics on surfaces is explained in Section 2.4.

\subsection{A Ridge Detector on Surfaces}

In this paper, we used the principal curvature of the surface to detect ridges and generate an intensity function $I$ that describes the texture manifold. We present the intensity function $I$ that gave the best result, however the algorithm applies for other choices of ridge detectors as well.

Let $\mathcal{S}$ be a smooth genus 0 surface and let $\kappa_{1}, \kappa_{2},\left|\kappa_{1}\right| \geq\left|\kappa_{2}\right|$ be the principal curvatures of $\mathcal{S}$ and $\mathbf{u}_{1}, \mathbf{u}_{2}$ their associated vector directions. We associate a fold with a line of low curvature in one direction with high curvature in the perpendicular direction. Let $\mathbb{S}$ be the unit circle and let $\mathbf{v}_{\theta} \in \mathbb{S}$. We define the probability to observe a fold at a given location $\mathbf{u} \in \mathcal{S}$ for a given orientation $\theta$ using a symmetric Von Mises distribution as

$$
I(\mathbf{u}, \theta)=n\left(\left|\kappa_{1}(\mathbf{u})\right|-\left|\kappa_{2}(\mathbf{u})\right|\right)^{-1} e^{\left(\left|\kappa_{1}(\mathbf{u})\right|-\left|\kappa_{2}(\mathbf{u})\right|\right)\left(\mathbf{u}_{\theta}^{t} \mathbf{u}_{1}\right)^{2}},
$$

where $n\left(\left|\kappa_{1}(\mathbf{u})\right|-\left|\kappa_{2}(\mathbf{u})\right|\right)$ is the normalization factor such that $\int_{\mathbb{S}} I(\mathbf{u}, \theta) d \theta=1$. Equation 4 can be seen as the equivalent of a Gaussian distribution for angles, with a maximum at $\mathbf{u}_{\theta}= \pm \mathbf{u}_{1}$ and where $\left|\kappa_{1}\right|-\left|\kappa_{2}\right|$ determines the spread around the maximums. 


\subsection{A Dictionary of Smooth Vector Fields to Model the Texture Manifold}

Unlike in images, it is not possible to hypothesize that the texture flow has a slope given by $v_{1}, v_{2}$ and then resample the image using these slope parameters as done in Equation 1. Instead, we use a base flow field $\mathbf{h}_{i}$. Let $\mathbf{h}_{i}(\mathbf{u})$ be the angle of the base flow field at a point $\mathbf{u} \in \mathcal{S}$. We can use $\mathbf{h}_{i}$ to resample $I$ as

$$
I_{i}(\mathbf{u}, \theta)=I\left(\mathbf{u}, \theta+\mathbf{h}_{i}(\mathbf{u})\right) .
$$

Then, we can apply a ridge detection to determine if $\mathbf{h}_{i}$ is a good hypothesis locally of the texture manifold.

The point of the algorithm is to generate several flow field $\mathbf{h}_{i}$, each with a different curvature. Each flow field $\mathbf{h}_{i}$ is generated by placing a source and a sink on $\mathcal{S}$ and then generating a smooth (singularity-free) completion between them. Specifically, let $\mathbf{s}_{i}=\left(\mathbf{s}_{i, 1}, \mathbf{s}_{i, 2}\right)$ be a source and a sink, respectively. A vector field is then completed on $\mathcal{S} / \mathbf{s}_{i}$ by finding the minimum of the following functional:

$$
\mathbf{h}_{i}=\operatorname{argmin}_{\mathbf{h}^{*}} \int_{\mathcal{S} / \mathbf{s}_{i}} \operatorname{curl}\left(\mathbf{h}^{*}\right)^{2}+\operatorname{div}\left(\mathbf{h}^{*}\right)^{2} d \mathcal{S} .
$$

We note that $\mathbf{h}_{i}$ is a unit vector field defined on $\mathcal{S}$. An example for a $\mathbf{h}_{i}$ is given in Figure 3(a). On this figure, the vector field fans in the vicinity of the singularity. To generate a full set of hypothesis $\mathbf{h}_{i}, i=1, \ldots, N$, we distribute sources and sinks uniformly on the cortical surface such that every location is offered a possibility to "fan" . An interesting property of our formulation in Equation 5 is that $\mathbf{h}_{i}$ can be seen as a baseline. The function $I_{i}(\mathbf{u}, 0)$ measures the likelihood that folds fans away from the singularity used to generate $\mathbf{h}_{i}$, as shown in Fig. 3(a), while the function $I_{i}(\mathbf{u}, \pi / 2)$ measures the likelihood that folds rotate around the same singularities, as shown in Fig. 3(b).

The streamline tracing algorithm works as follows. Once it is determined that $\mathbf{h}_{i}$ is a locally optimal hypothesis an initial streamline is launched with an angle of $\alpha$ with $\mathbf{h}_{i}$ (for example $\alpha=\pi / 2$ ). We then follow the flow given by the function $\mathbf{h}_{i}+\alpha$ over the entire region for which $\mathbf{h}_{i}$ is the optimal hypothesis. Examples of streamlines generated by this process are shown in Figure 3(c).

\subsection{Relaxation Labeling of Tangential and Normal Curvature}

Till now we have described an algorithm to generate flow fields $\mathbf{h}_{i}$ on a surface $\mathcal{S}$. In this section, we describe how to select, from $\mathbf{h}_{i}$, an optimal assignment that best matches the flow field oberved on a surface. Let $H^{*}(\mathbf{u}, \theta) \in\left\{\mathbf{h}_{1}, \ldots, \mathbf{h}_{n}\right\}$. We use relaxation labeling [8] to determine which of the possible flows $\mathbf{h}_{i}$ offers the best local fit to the fold lines of the cortical surface. Relaxation labeling is a framework to find the statistical mode of a distribution given general constraints to be satisfied. Let $p_{i}(\mathcal{S}, \theta)$ be the probability that hypothesis $\mathbf{h}_{i}(\mathcal{S}, \theta)$ has the highest support at any given location. Here, we interpret $\mathbf{h}_{i}(\mathcal{S}, \theta)$ has the hypothesis given by $\mathbf{h}_{i}$ with a flow field with initial angle $\theta$. The $p_{i}(\mathcal{S}, \theta)$ forms a probability space, such that $p_{i}(\mathcal{S}, \theta)>0$ and $\sum_{i=1}^{N} p_{i}(\mathcal{S}, \theta)=1$ everywhere. 


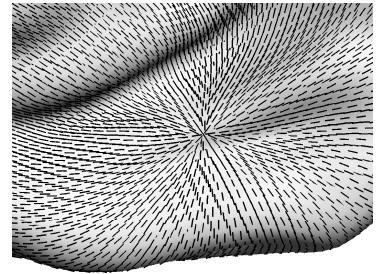

(a) Vector field in the vicinity of a sink

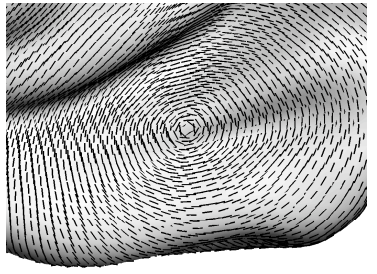

(b) Vector field curling around a singularity

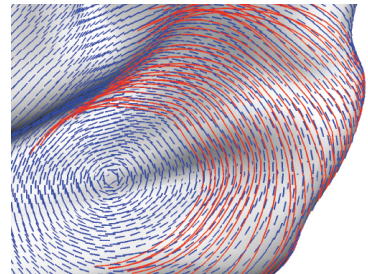

(c) Streamlines inferred using curvatures and initial angles from Fig.3(b).

Fig. 3.

Relaxation labeling updates the $p_{i}$ in order to maximize the local fit while making sure that each label is well supported by its neighbors. In our case, the optimal solution should (1) maximize the function $f$ in Eq. 3 and (2) find solutions that are supported over a large spatial region.

To determine the solutions that have a large support on the manifold, we minimize the gradient of $p_{i}$ along both $\mathcal{S}$ and $\theta$ as follows. Let $\mathbf{p}_{i}^{\prime}$ be the resampled value of $\mathbf{p}_{i}$ along $\mathbf{h}_{i}$ as

$$
\mathbf{p}_{i}^{\prime}(\mathbf{u}, \theta)=\mathbf{p}_{i}\left(\mathbf{u}, \theta+\mathbf{h}_{i}\right) .
$$

Then, we regularize the assignment $\mathbf{p}_{i}$ by minimizing the spatial and orientation gradient of $\mathbf{p}_{i}^{\prime}$ as

$$
U_{\text {reg }}\left(p_{i}\right)=\left(\left\|\nabla_{\mathcal{S}} p_{i}^{\prime}\right\|^{2}\right) \circ \mathbf{h}_{i}^{-1}+\lambda_{1}\left\|\nabla_{\theta} p_{i}\right\|^{2},
$$

where $\circ \mathbf{h}_{i}^{-1}$ is used as a short-hand to mean that we should resample $\left(\left\|\nabla_{\mathcal{S}} p_{i}^{\prime}\right\|^{2}\right)$ along the original $\theta$ orientation.

A relaxation labeling framework is then used to maximize the following functional

$$
\mathbf{p}^{*}=\operatorname{argmax}_{\mathbf{p}} \sum_{i} \int_{\mathcal{S} \times \mathbb{S}}-U_{r e g}\left(p_{i}\right)+\lambda_{2} p_{i} f_{i}+\lambda_{3}\left\|p_{i}\right\|^{2} d \mathcal{S} \wedge d \theta
$$

where the $\left\|p_{i}\right\|^{2}$ term is added to make the relaxation labeling scheme converge to an unambiguous labeling, i.e., $p_{i}=\{0,1\}$, as explained in [8].

Finally, there exist multiple methods to maximize Equation 9. One of the simplest is to use gradient ascent [8], which for any $p_{i}$ gives

$$
\frac{\partial p_{i}}{\partial t}=-\left(\Delta_{\mathcal{S}} p_{i}^{\prime}\right) \circ \mathbf{h}_{i}-\lambda_{1} \Delta_{\theta} p_{i}-\lambda_{2}\left\|\nabla f^{t} \mathbf{n}_{i}\right\|+\lambda_{3} p_{i} .
$$

\subsection{Statistical Tests over Curve Length}

We now estimate how curve length is affected by the presence of external factors. First, we launch streamline flows in multiple directions at every location on the surface. In practice we are not given trajectories, but rather an optimal 
assignment $H^{*}$ given by the probabilities $p_{i}$. Let $\gamma(l)$ be a curve parametrized by arc-length and $\mathbf{t}$ the tangent of $\gamma(l)$. The curvature of $\gamma(l)$ (and hence its trajectory) is determined from the assignment $p_{i}$ as

$$
\frac{\partial}{\partial l} \mathbf{t}=\sum_{i} p_{i}(\gamma, \mathbf{t})\left[\frac{\partial}{\partial l} \mathbf{h}_{i}\right] .
$$

In practice, we use a first order Euler method to trace streamlines on surfaces. We take a small step in a given direction, then we compute the change in angle that is prescribed by Equation 11 given the current position and the given angle. Curve length is then measured explicitly as

$$
L(\gamma)=\int_{[-T, T]} \sqrt{\left\|\frac{\partial \gamma}{\partial s}\right\|^{2}} d s .
$$

Let $L(\gamma(\mathbf{u}, \theta))$ be the length of the curve started at $\mathbf{u} \in \mathcal{S}$ at a given orientation $\theta$. The reader may realize that we have previously defined $\|\mathbf{t}\|=1$ and thus $L(\gamma)=$ $2 T$. However, Equation 12 becomes useful when we use a shape diffeomorphism onto a template space. Assume that there are $n$ surfaces $\mathcal{S}_{l}, l=1, \ldots, n$ with a set of diffeomorphisms that map these surfaces onto a template average $\overline{\mathcal{S}}$ :

$$
\phi_{l}: \mathcal{S}_{l} \rightarrow \overline{\mathcal{S}}
$$

Then, a random field can be defined by measuring the length of the curve $\gamma$ when mapped using $\phi_{l}$ onto $\mathcal{S}_{l}$ as $L\left(\phi_{l}(\gamma(\mathbf{u}, \theta))\right)$. We then assume that the logarithm of curve length follows a Gaussian distribution, thus allowing us to define a Gaussian random field on $\mathcal{S} \times \mathbb{S}$. Some regularization and random field theory is then used to correct for multiple comparisons (see [9]).

\section{Results}

To present result, we first illustrate the algorithm by generating curves using a flow field $\mathbf{h}_{i}$. We use a template surface and then generate a full estimate of the curvature of the texture manifold using Equation 11. Several streamlines are launched in the direction of sulcal lines, as shown in Fig. 4. Observe that these streamlines bend and fan to follow the folds, qualitatively demonstrating the accuracy of the recovered curvature field.

We used the OASIS database [7] to determine if our method could find curves whose length is significantly correlated with the presence of mild cognitive impairment in Alzheimer's disease (AD). The OASIS database consists of 97 healthy subjects and 92 subjects (aged 60 and above) affected with mild and very-mild dementia. We used the extraction pipeline of [3], which produces one mid-surface representation of the gray-matter cortical sheet, and obtained mappings $\phi_{l}$ for each surface onto a common template average. Once this mapping was found, we computed the average surface of the entire population and used this average surface to compute curvature estimates using Equation 11. 


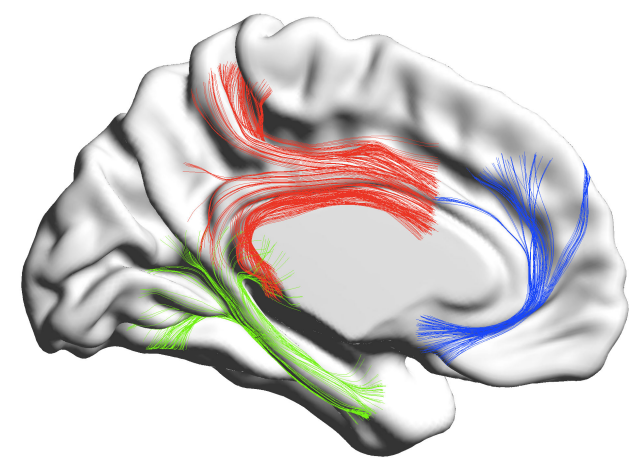

Fig. 4. Examples of streamlines of length $8 \mathrm{~cm}$ launched in directions that are tangent to sulcal lines. The streamlines bend and fan to follow the fold patterns. There are multiple gyrii fanning away in the vicinity of the blue lines.

The lengths of the computed curves were then tested to see if any significant correlations could be found with the presence of mild cognitive impairment in the OASIS database. The results are shown in Figure 6. The white streamlines indicate a significant dilation while the green streamlines show a significant contraction. These results show that the main shape differences are both perpendicular and parallel to the fold direction. The contraction is in the temporal lobe, which is known to be affected with the presence of Alzheimer's disease. The dilation in the temporal lobe is probably due to atrophy of the hippocampus.

The most significant result is that our method is able to identify a stretching in length of the Cingulate gyrus below Praecuneus area and the area below the sup frontal cortex in the left hemisphere. We also performed a statistical test on the surface area [10] and these results are shown in Fig. 5. Whereas this test detects the same region (below the Cingulate gyrus) it does not reveal if the larger area comes from a wider or a longer sulcus.

Another interesting aspect of our method is that it integrates deformation along a very narrow streamline, permitting the use of an anisotropic kernel for regularization. Thus, the results using surface area were not significant in the region below the Cingulate cortex after correction for multiple comparison using Random Field correction [9]. However, the use of anisotropic filters produced significant results in both the temporal lobe and the Cingulate cortex, as shown in Fig. 6. The threshold for significance are $|t|>4.25$ using Random Field Theory and $|t|>4.31$ using a permutation test with 50000 permutations. The peak values of the three regions shown in Fig. 6 were above the permutation test threshold and the $p$ values reported in the caption of Figure 6 are computed using Random Field correction [9].

Overall these results show that it is possible using directional filters to gain an insight into the process that leads to brain deformations. Thus, wether the stretch in length is due to structural connectivity loss in the white matter still 


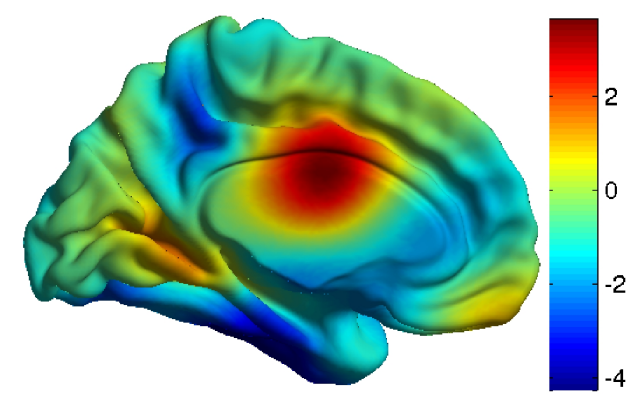

Fig. 5. Comparisons of the surface area of patients with mild cognitive impairment (MCI) and healthy subjects. A statistical t-Test (indicated by the colorbar) shows that the region below the Cingulate cortex has a larger surface area in the group with MCI. However, the t-Map does not reveal whether the larger area comes from a wider or a longer sulcus.

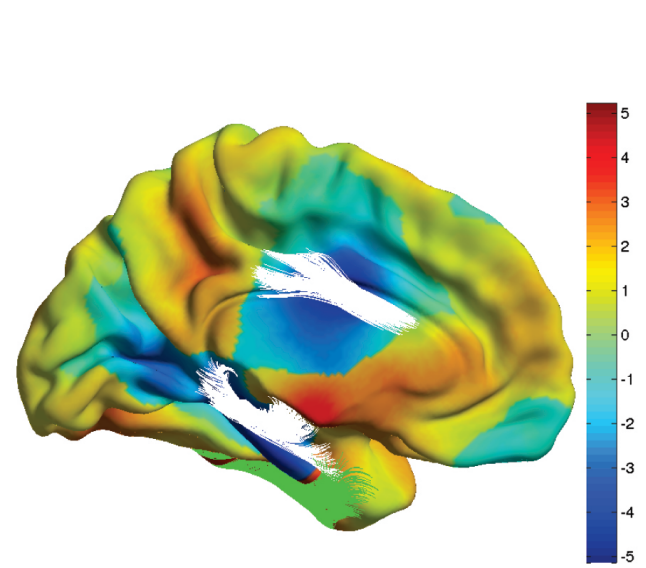

(a) Saggital cut of the left hemisphere. The t-Test shows that the larger area in Fig. 5 is due to a longer Cingulate gyrus in the group with MCI.

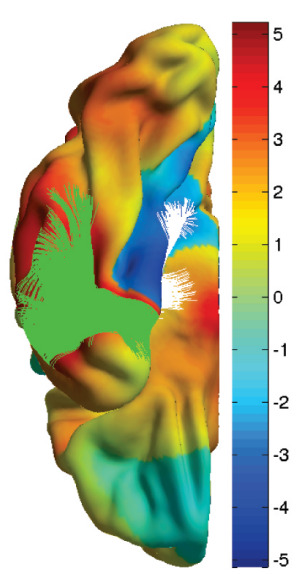

(b) Bottom view

Fig. 6. Positive values shows significant dilation in the AD group. White streamlines show a significant dilation of the curve length $(p<0.0015$ on top of the hyppocampal gyrus and $p<0.01$ on the cingulate gyrus) and green streamlines in the temporal lobe show a significant contraction $(p<0.0007)$. Color indicates the maximum in absolute value of the $t$-test over all possible orientations. 
needs to be investigated, but our findings are corroborated by a visual inspection of shape differences.

\section{Conclusion}

We have described a method to perform a dense statistical analysis of the cerebral cortex using curve-based morphometry. Our method departs from other statistical methods, e.g., those based on shape diffeomorphisms. We define a manifold of curves on the cerebral cortex and then use statistics on curve length to examine shape changes. We obtain novel results concerning the nature of changes in cortical fold patterns in subjects with mild cognitive impairment.

\section{References}

1. Fischl, Sereno, Dale: Cortical surface-based analysis. II: Inflation, flattening, and a surface-based coordinate system. Neuroimage 9(2) (1999) 195-207

2. Leporé, N., Brun, C., Pennec, X., Chou, Y., Lopez, O., Aizenstein, H., Becker, J., Toga, A., Thompson, P.: Mean template for tensor-based morphometry using deformation tensors. In: Proceedings of the 10th international conference on Medical image computing and computer-assisted intervention, Springer-Verlag (2007) 826-833

3. Lyttelton, O., Boucher, M., Robbins, S., Evans, A.: An unbiased iterative group registration template for cortical surface analysis. Neuroimage 34(4) (2007) 15351544

4. Worsley, K.: Testing for signals with unknown location and scale in a chi^ 2 random field, with an application to fMRI. Advances in Applied Probability 33(4) (2001) $773-793$

5. Chung, M., Worsley, K., Evans, A.: Tensor-based brain surface modeling and analysis. Computer Vision and Pattern Recognition, 2003. Proceedings. 2003 IEEE Computer Society Conference on 1 (2003) 467-473

6. Ben-Shahar, O., Zucker, S.: The perceptual organization of texture flow: A contextual inference approach. PAMI 25(4) (2003) 401-417

7. Marcus, D., Wang, T., Parker, J., Csernansky, J., Morris, J., Buckner, R.: Open Access Series of Imaging Studies (OASIS): Cross-sectional MRI Data in Young, Middle Aged, Nondemented, and Demented Older Adults. Journal of Cognitive Neuroscience 19(9) (2007) 1498-1507

8. Hummel, R., Zucker, S.: On the foundations of relaxation labeling processes. In: Readings in computer vision: issues, problems, principles, and paradigms, Morgan Kaufmann Publishers Inc. (1987) 605

9. Adler, R., Taylor, J.: Random fields and geometry. Springer New York (2007)

10. Chung, M., Worsley, K., Robbins, S., Paus, T., Taylor, J., Giedd, J., Rapoport, J., Evans, A.: Deformation-based surface morphometry applied to gray matter deformation. NeuroImage 18(2) (2003) 198-213 